\title{
A First-Order Cyclostationarity Based Energy Detection Approach for Non-Cooperative Spectrum Sensing
}

\author{
Zhengwei Lu, Yi Ma, and Rahim Tafazolli \\ CCSR, The University of Surrey, Guildford \\ e-mail:\{z.lu, y.ma, r.tafazolli\}@ surrey.ac.uk
}

\begin{abstract}
Spectrum sensing is one of key enabling techniques to advanced radio technologies such as cognitive radios and ALOHA. This paper presents a novel non-cooperative spectrum sensing approach that can achieve a good trade-off between latency, reliability and computational complexity. Our major idea is to exploit the first-order cyclostationarity of the primary user's signal to reduce the noise-uncertainty problem inherent in the conventional energy detection approach. It is shown that the proposed approach is suitable for detecting the primary user's activity in the interweave paradigm of cognitive spectrum sharing, while the active primary user is periodically sending training sequence. Computer simulations are carried out for the typical IEEE 802.11g system. It is observed that the proposed approach outperforms both the energy detection and the secondorder cyclostationarity approach when the observation period is more than 10 frames corresponding to $0.56 \mathrm{~ms}$.
\end{abstract}

\section{INTRODUCTION}

Spectrum sensing is one of key techniques to enable advanced radio technologies such as cognitive spectrum sharing and random multiple access methodology (e.g. ALOHA), and thus has received increasing attentions in the last decade [1]. The main concept is to monitor user activity within a certain frequency band through employment of advanced signal processing techniques at a receiver. Key metrics used to benchmark the reliability of a spectrum sensing approach includes probability of detection (PD) and probability of false alarm (PFA) [2]. Moreover, a promising spectrum sensing approach should offer small latency (mainly the time duration of observation), low computational complexity [3].

State of the art spectrum sensing approaches can be classified into two categories, i.e. cooperative spectrum sensing and non-cooperative spectrum sensing. The former one can be regarded as a cooperative communication aided technique, where geographically distributed cognitive devices cooperate with each other through message passing to gain the spatial diversity [4]. Nevertheless, each cognitive device still employs non-cooperative spectrum sensing approaches such as energy detection [5], second-order cyclostationarity detection [6], or others to produce the initial result.

In the category of non-cooperative spectrum sensing, energy detection is the simplest and widely used approach that offers linear computational complexity and small latency. Its main shortcoming is the well-known noise uncertainty problem, which results in energy detection approach failed in the low signal-to-noise ratio (SNR) scenario [7]. Second-order cyclostationarity is recognized as the most reliable approach due to its immunity to the noise uncertainty [8]. On the other hand, the second-order cyclostationarity approach requires square computational complexity, and a considerably long data-record-length (large latency) for the convergence. Other methods include, for example, the matched filtering approach [9] that requires fair timing synchronization before the spectrum sensing ${ }^{1}$, and the eigenvalue decomposition approach [10] that requires multiple receive-antennas at the cognitive device. More recently, a wavelet-based edge detection approach has been proposed and proved to be effective for the wideband spectrum sensing [11].

Consider one of typical wireless scenarios where a cognitive device equipped with a single antenna is performing noncooperative spectrum sensing. The cognitive device is not synchronized with the primary system. Is there existing an efficient spectrum sensing approach that can offer a good trade-off between reliability, latency and complexity? This paper is motivated by the above question, and presents a novel first-order cyclostationarity based approach. Our major idea is based upon a practical condition that, in most of primary systems, the transmitter sends periodic training sequences for the purpose of synchronization and channel tracking. This distinctive feature yields the first-order cyclostationarity, which can be used to reduce the noise-uncertainty problem so that a reliable energy detector can be achieved at low SNR environment. Therefore, our approach is quite different with other existing first-order cyclostationarity based approaches, for example [12] and [13], where it utilizes the cyclic frequencies of the received signal to perform spectrum sensing. In order to test the proposed approach, computer simulations are carried out for the typical IEEE $802.11 \mathrm{~g}$ system. It is observed that the proposed approach outperforms both the energy detection and the second-order cyclostationarity approach in terms of the reliability when the observation period is more than 10 frames corresponding to $0.56 \mathrm{~ms}$.

\section{SySTEM MODEL}

Fig. 1 depicts the smallest system for studying the noncooperative spectrum sensing, where a cognitive mobile device in the secondary network wants to opportunistically access the primary network's spectrum in the interweave manner. It is assumed that the primary transmitter, if active, would periodically send training sequences/preambles for the purpose

\footnotetext{
${ }^{1}$ This is not a reasonable assumption in practice.
} 


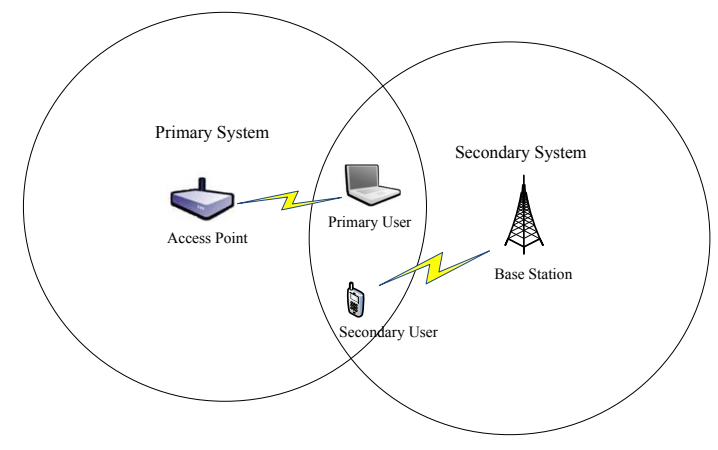

Fig. 1. An example of the smallest system for studying the spectrum sensing.

of synchronization and channel estimation. The cognitive device senses the primary user's activity for each operating band.

Denote $\mathbf{p} \triangleq\left[\mathrm{p}_{1}, \mathrm{p}_{2}, \cdots, \mathrm{p}_{K},\right]^{T}$ to be the training block, and $\mathbf{s}_{i} \triangleq\left[\mathrm{s}_{i, 1}, \mathrm{~s}_{i, 2}, \cdots, \mathrm{s}_{i, M}\right]^{T}$ to be the information-bearing symbol block, where $K, M$ stands for the block size, and $i$ for the block index. The signal sent by the primary transmitter can be expressed as a sequence $\left(x_{1}, x_{2}, \cdots\right) \triangleq$ $\left(\mathbf{p}^{T}, \mathbf{s}_{0}^{T}, \mathbf{p}^{T}, \mathbf{s}_{1}^{T}, \cdots\right)$. We assume that the cognitive device knows the standard of primary system, and thus has the knowledge about the symbol rate, the preamble duration, and the block duration. Then, the cognitive device can perform an appropriate analog-to-digital conversion, and has the preprocessing digital waveform as

$$
\mathrm{y}_{n}=\left\{\begin{array}{l}
v_{n}, \quad H_{0} \\
\sum_{l=0}^{L_{h}-1} h_{l} x_{n-l}+v_{n}, H_{1}
\end{array}\right.
$$

where $h_{l}$ stands for the $l$ th tap of the multipath channel, $L_{h}$ for the channel length, and $v_{n}$ for the additive white Gaussian noise (AWGN) with zero mean, $H_{0}$ and $H_{1}$ donate the hypothesis for primary signal presence and absence, respectively. The multipath channel, in this paper, is considered as static. This is because our simulation results have shown that the proposed approach can achieve a satisfactory performance within a very short observation time. In the low-mobility scenario, $h_{l}$ does not vary considerably within the observation time. Then, the objective of spectrum sensing is to make a binary decision in $\left(H_{0}, H_{1}\right)$ through performing certain signal processing on $\mathrm{y}_{n}$.

\section{First-Order Cyclostationarity Based Energy DETECTION}

\section{A. Detection Algorithm}

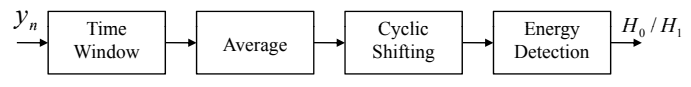

Fig. 2. Block diagram of the proposed approach

Fig. 2 shows the block diagram of the proposed detector. To enable the detector, the received signal at the secondary

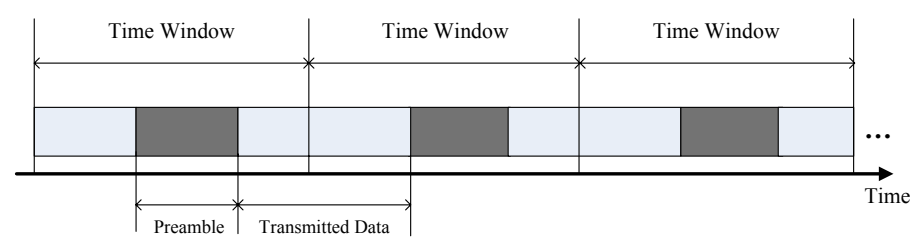

Fig. 3. Time window operation without time synchronization

user's receiver is firstly separated into several sections by a time window, $T W 1$, which is shown Fig. 3. One should be noted that the time synchronization does not be required in this stage. The first-order statistics is then performed in the above signal. Next, in order to find the peak portion of the signal, another time window, $T W 2$, is used to perform cyclic shifting operation. When the peak portion is located, the conventional energy detection scheme will be followed.

In detail, the first-order statistics of the received signal after the time window can be expressed by

$$
\begin{aligned}
r_{n} & =\frac{1}{D} \sum_{d=0}^{D-1} y_{n+d N_{1}} \\
& =\frac{1}{D} \sum_{d=0}^{D-1}\left(\sum_{l=0}^{L_{h}-1} h_{l} x_{n+D N_{1}-l}+v_{n+d N_{1}}\right)
\end{aligned}
$$

where $n \in\left[0, N_{1}-1\right], D$ donates the number of sections which are separated by $T W 1$ in the received signal, $N_{1}$ indicates the window length of $T W 1$. Since the cognitive device knows the standard of the primary system, the $N_{1}$ is equal to the frame length of the primary system. Hence, the parameter $D$ can reflect the observation time, indirectly. Because $h_{l}$ does not vary considerably within the observation time, (2) can be following as

$$
r_{n}=\sum_{l=0}^{L_{h}-1} h_{l} \sum_{d=0}^{D-1} \frac{x_{n+d N_{1}-l}}{D}+\sum_{d=0}^{D-1} \frac{v_{n+d N_{1}}}{D}
$$

By the definition of the primary signal sequence, $\left(x_{1}, x_{2}, \cdots\right) \triangleq\left(\mathbf{p}^{T}, \mathbf{s}_{0}^{T}, \mathbf{p}^{T}, \mathbf{s}_{1}^{T}, \cdots\right)$, we are able to rewrite (3) into two parts: one is for the preamble part of the $r_{n}$, the other is for the transmitted data part of the $r_{n}$

$$
r_{n}=\sum_{l=0}^{L_{h}-1} h_{l} \sum_{d=0}^{D-1} \frac{\mathrm{p}_{k+d N_{1}-l}}{D}+\sum_{d=0}^{D-1} \frac{v_{n+d N_{1}}}{D}
$$

and

$$
r_{n}=\sum_{l=0}^{L_{h}-1} h_{l} \sum_{d=0}^{D-1} \frac{\mathrm{s}_{(d, m)-l}}{D}+\sum_{d=0}^{D-1} \frac{v_{n+d N_{1}}}{D}
$$

where $1 \leq k \leq K, 1 \leq m \leq M$ and $d$ equal to $i$ to indicate the block index. Due to both the noise and the information symbol have zero mean, as $D \rightarrow \infty$, it is clearly that

$$
\sum_{d=0}^{D \rightarrow \infty} \frac{\mathrm{s}(d, M)-l}{D}=0, \quad \sum_{d=0}^{D \rightarrow \infty} \frac{v_{n+d N_{1}}}{D}=0
$$


On the other hand, the training sequence in each frame is fixed, the average operation can not affect the power of training sequence

$$
\sum_{d=0}^{D \rightarrow \infty} \frac{\mathrm{p}_{k+d N_{1}-l}}{D}=\mathrm{p}_{k-l}
$$

Therefore, we can obtain

$$
\mathbf{r}_{\mathbf{n}}=\left[\left(\sum_{l=0}^{L_{h-1}} h_{l} \mathrm{p}_{k-l}\right), 0,0, \ldots, 0\right]^{T}, 1 \leq k \leq K
$$

This result implies that the power of the noise and the information symbol can be reduced by increasing the observation time, while the training sequence is kept. A peak will thus appear in the $\mathbf{r}_{\mathbf{n}}$. If we perform the energy detection in the such peak portion, the noise uncertainty will no longer be a problem.

\section{B. Timing Offset}

Since the secondary system usually does not synchronize with the primary system, the training block may locate in anywhere within the $\mathbf{r}_{\mathbf{n}}$ $\left(\right.$ i.e. $\left.\mathbf{r}_{\mathbf{n}}=\left[0,0, \ldots,\left(\sum_{l=0}^{L_{h-1}} h_{l} \mathrm{p}_{k-l}\right), 0,0, \ldots, 0\right]^{T}\right)$.

It means that the peak may also locate in anywhere within the $\mathbf{r}_{\mathbf{n}}$. In order to find this peak, we use another time window, $T W 2$, to perform cyclic shifting in the $\mathbf{r}_{\mathbf{n}}$, where the window length is $N_{2}$ and is equal to the training block size. We perform the operation by

$$
\begin{array}{r}
T W 2_{\tau}=\sum_{n=0}^{N_{2}-1}\left|r_{n+\tau}\right|, \quad \tau \in\left[0, N_{1}-N_{2}-1\right] \\
T W 2_{\tau}=\sum_{n=0}^{N_{1}-\tau-1}\left|r_{n+\tau}\right|+\sum_{n=0}^{N_{2}-\left(N_{1}-\tau\right)-1}\left|r_{n}\right|, \\
\tau \in\left[N_{1}-N_{2}, N_{1}-1\right]
\end{array}
$$

where $\tau$ stands for the timing offset.

Base on (6) and (7), as $D \rightarrow \infty$, the power of the noise and the information symbol will be close to zero while the training block is kept. Thus, the peak can be found by searching the maximum $T W 2_{\tau}$

$$
\max \left(T W 2_{\tau}\right) \rightarrow \tau_{\mathrm{p}}
$$

where $\tau_{\mathrm{p}}$ represents the starting point of the peak portion in the $\mathbf{r}_{\mathbf{n}}$. When this peak is located, we will follow the conventional energy detection. The test statistic for energy detection is

$$
\text { Test }=\sum_{n=\tau_{\mathrm{p}}}^{\tau_{\mathrm{p}}+N_{2}-1}\left|r_{n}\right|^{2}
$$

\section{Energy Detection}

We define the receiver input SNR as

$$
\mathrm{SNR} \triangleq \frac{P_{s}}{P_{n}}
$$

where $P_{s}$ represents the signal power, $P_{n}$ donates the noise power. Base on our proposed approach, it is obviously that the noise power can be reduced linearly by increasing the observation time (i.e. $\frac{P_{n}}{D}$ ), while the training block power is kept and shows as a peak in the signal. Hence, the SNR in the such peak can be expressed by

$$
\mathrm{SNR}_{\text {peak }}=\frac{P_{s}}{P_{n} / D}=\frac{D P_{s}}{P_{n}}
$$

where $\mathrm{SNR}_{\text {peak }}$ represents the SNR in the peak portion of the signal. This equation implies that the $\mathrm{SNR}_{\text {peak }}$ can be improved by increasing the observation time.

According to [5] and [14], the means and variances of the test statistic under hypothesis $H_{i}(i=0,1)$ are

$$
\mu_{0}=\frac{P_{n} N_{2}}{D}, \quad \sigma_{0}^{2}=\frac{2 P_{n}^{2} N_{2}}{D^{2}}
$$

and

$\mu_{1}=\frac{P_{n} N_{2}\left(S N R_{\text {peak }}+1\right)}{D}, \quad \sigma_{1}^{2}=\frac{2 P_{n}^{2} N_{2}\left(2 S N R_{\text {peak }}+1\right)}{D^{2}}$

The PFA and the PD can be expressed by

$$
P_{f a}=Q\left(\frac{T h-\mu_{0}}{\sigma_{0}}\right)
$$

and

$$
P_{d}=Q\left(\frac{T h-\mu_{1}}{\sigma_{1}}\right)
$$

where $Q(x)$ represents the Q-function, $Q(x) \triangleq$ $\frac{1}{\sqrt{2 \pi}} \int_{x}^{\infty} e^{-\frac{u^{2}}{2}} d u, T h$ is the decision threshold. One should be noted that (18) is for the AWGN channel. The PD for Rayleigh, Nakagami, and Rician fading channels have been derived in [15].

In the most real environment, in actually, $P_{n}$ is estimated by a radiometer. An error is therefore usually exist

$$
\left(1-\epsilon_{1}\right) P_{n} \leq \hat{P}_{n} \leq\left(1+\epsilon_{2}\right) P_{n}
$$

where $0 \leq \epsilon_{1}<1$ and $\epsilon_{2} \geq 0$. As shown in [14], the peak-topeak noise uncertainty $U$ can be defined as

$$
U \triangleq \frac{1+\epsilon_{2}}{1-\epsilon_{1}}
$$

For the conventional energy detection approach, it has been shown that the noise uncertainty results in the detector failed when the SNR lower than $U-1$ [7][14], no matter how long the observation time is used. In our proposed detector, however, we utilize the first-order cyclostationarity of the received signal to reduce the noise power, which is corresponding to improve the SNR. Therefore, with a certain observation time, our detector can always be achieved. 


\begin{tabular}{|c|c|}
\hline Parameters & Value \\
\hline $\mathrm{N}_{\mathrm{ftt}}$ & 64 \\
\hline $\mathrm{N}_{\mathrm{cp}}$ & 16 \\
\hline Bandwidth & $20 \mathrm{MHz}$ \\
\hline Number of OFDM blocks per frame & 10 \\
\hline
\end{tabular}

Fig. 4. System parameters set-up

At last, the decision threshold can be finally obtained by

$$
T h=U\left[\mu_{0}+\sigma_{0} Q^{-1}\left(P_{f a, d e s}\right)\right]
$$

where $P_{f a, d e s}$ donates a desired PFA.

\section{Simulation Results and Discussion}

In this section, the proposed spectrum sensing scheme is evaluated through computer simulations. An orthogonal frequency-division multiplexing (OFDM)-based IEEE 802.11g system [16] was chosen as the primary system. Each OFDM block contains 64 sub-carriers with cyclic prefix (CP) length of 16. The sub-carriers designate as follows: 48 data, 4 pilot and 12 guard. One OFDM block during is $4.0 \mu \mathrm{s}$ (including $\mathrm{CP})$ and one OFDM frame contains 10 OFDM blocks. The system parameters set-up is shown in Fig.4.

The channel model adopted in the simulations are the WINNER channel model under scenario A1 with Line-ofSight (LOS) condition [17]. The mobile station speed sets up at $1.0 \mathrm{~m} / \mathrm{s}$. The maximum Doppler shift is therefore at $8.167 \mathrm{~Hz}$ for a carrier frequency of $2.450 \mathrm{GHz}$. All curves are averages over 2000 independent experiments.

In Fig.5 and Fig.6, we compare the performance of the proposed detector with the conventional energy detector, where different observation time were chosen and $P_{f a, d e s}=10 \%$. In Fig.5, the observation time in here is equal to one frame duration of the primary signal. The proposed detector is thus like a conventional energy detector. Since the detector is just measuring part of the received signal, the performance of the proposed detector is slightly worse than the conventional energy detector. But with the observation time increasing, in Fig.6, the performance of the proposed detector is improved significantly, while the conventional energy detector is nearly keeping the same level due to the noise uncertainty. In this paper, we define that if the $P_{d}$ is equal or large than $90 \%$, then we claim that the primary signal is present. In Fig.7 the SNR requirement for successful detection of the primary signal under different observation time for the proposed detector and conventional energy detector is plotted. From this figure, we can see that increasing observation time can not further help the traditional energy detector to improve its performance due to the noise uncertainty. However, the proposed detector can 'break' the problem to improve the performance by utilizing the first-order cyclostationarity of the received signal. In actually, we do not resolve the noise uncertainty problem since it is a fundamental limit for energy detector. But we reduce the problem by another way.

The performance comparison between the proposed detector and the traditional second-order based cyclosationarity detec-

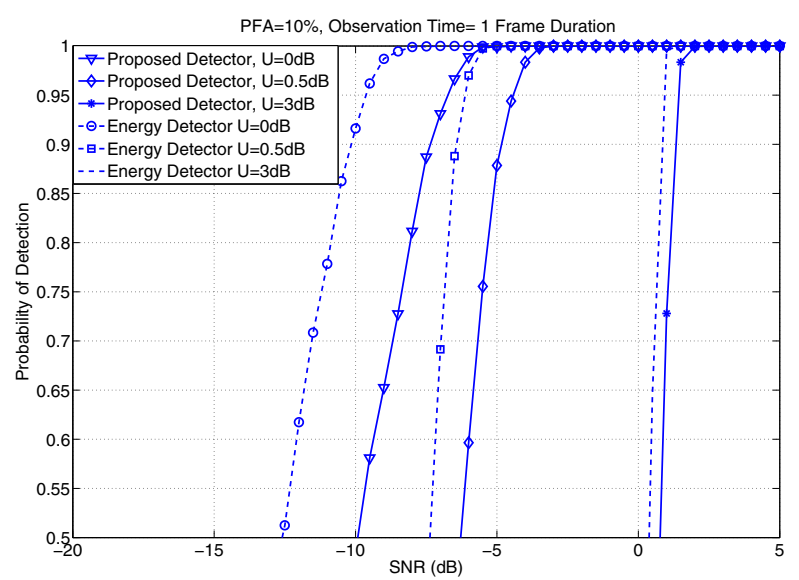

Fig. 5. Performance comparison between the proposed detector and the traditional energy detector, observation time $=1$ frame duration

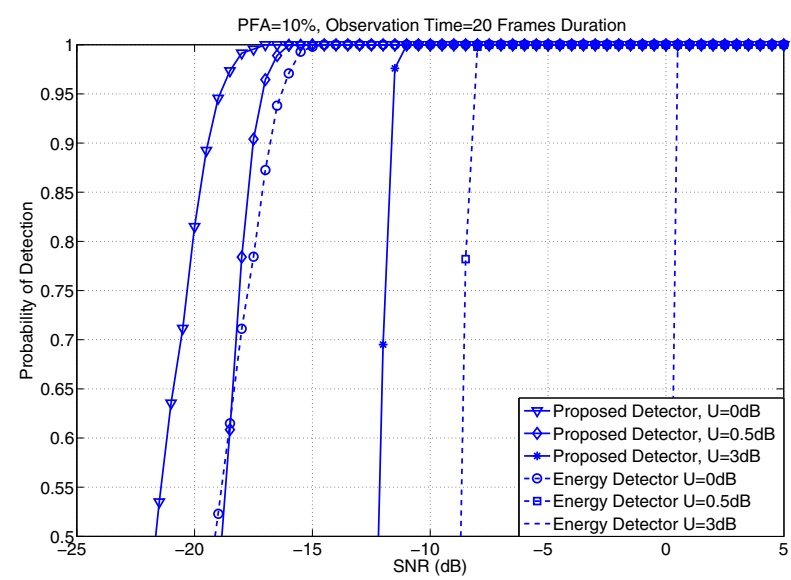

Fig. 6. Performance comparison between the proposed detector and the traditional energy detector, observation time $=20$ frames duration

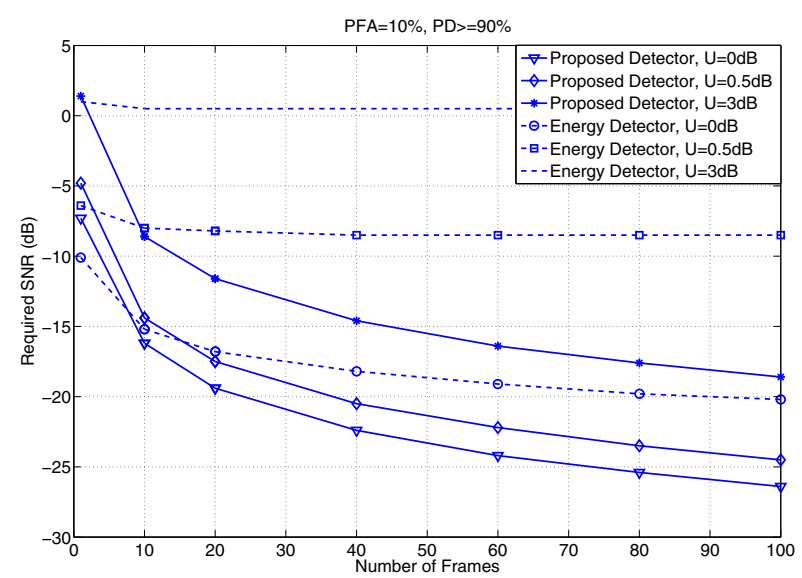

Fig. 7. SNR requirement for successful detection of the primary signal under different observation time for the proposed detector and the traditional energy detector 
tor is plotted in Fig.8, where the observation time is equal to 20 frames duration. For the second-order based cyclosationarity detector, we use the time-domain test algorithm in [18] to detect whether the primary signal is present or not. Since the CP is periodically sending, we fix $\tau_{a c}=64$, where $\tau_{a c}$ stands for the lag of the autocorrelation function of the received signal, and a length-2049 Kaiser window with $\beta$ parameter of 10 was used. Although both detectors are cyclostationarity based, they are quite different. Our proposed detector is utilizing the firstorder cyclostationarity to help the energy detector to reduce the noise uncertainty problem. A linear computational cost is therefore kept. The second-order cyclosationarity detector, however, requires square computational cost and it utilizes the cyclic frequencies of the received signal to detect the primary signal. From Fig.8, it has shown that even with $3 \mathrm{~dB}$ noise uncertainty, the performance of the proposed detector is better than the second-order cyclosationarity detector for successful detection of the primary signal. This result implies that the proposed detector requires less observation time than the traditional second-order cyclosationarity detector to achieve a desired performance. Fig.9 illustrates the SNR requirement for successful detection of the primary signal under different observation time for the proposed detector and the secondorder cyclosationarity detector. From this figure, we can see that the SNR requirement for the proposed detector with $3 \mathrm{~dB}$ noise uncertainty is very close to the second-order cyclosationarity detector, when the observation time is equal to 10 frames duration. This is because that the noise power can not reduce significantly within a very short observation time. For example, the observation time is equal or less than 10 frames duration. The performance of the proposed detector is still limited by the noise uncertainty problem. At last, the simulation results have shown that proposed detector outperforms both the conventional energy detector and the second-order cyclostationarity detector in terms of the reliability when the observation period is more than 10 frames corresponding to $0.56 \mathrm{~ms}$.

\section{Conclusion And Outlook}

In this paper, a first-order cyclostationarity based energy detection approach was proposed. The major idea is to exploit the first-order cyclostationarity of the primary user's signal to reduce the noise-uncertainty problem inherent in the conventional energy detection approach. The detector can be used in pilot based communication systems. Simulation results have shown the proposed approach outperforms both the energy detection and the second-order cyclostationarity approach when the observation period is more than 10 frames corresponding to $0.56 \mathrm{~ms}$. In the next stage, we will extend our approach into multiband scenarios and consider how to estimate the signal that if it comes from the primary system or other secondary systems by exploitation the cyclostationarity of the received signal.

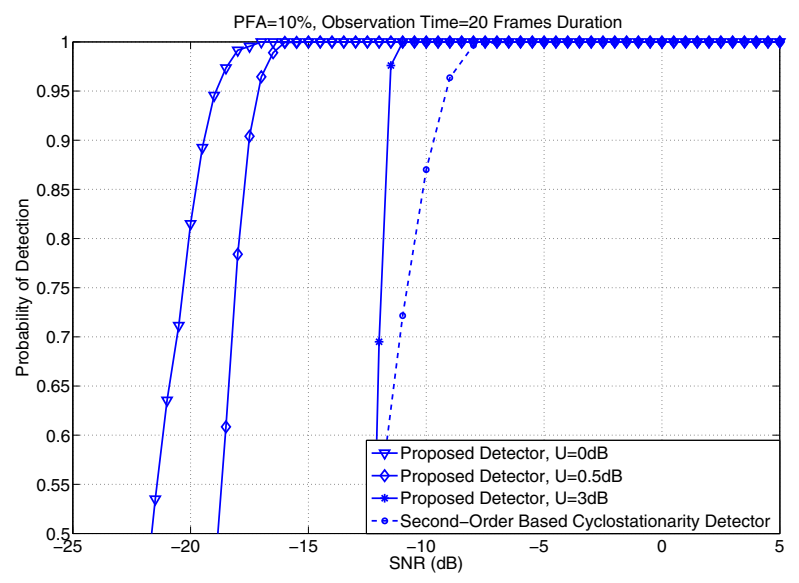

Fig. 8. Performance comparison between the proposed detector and the second-order based cyclostatinarity detector, observation time $=20$ frames duration

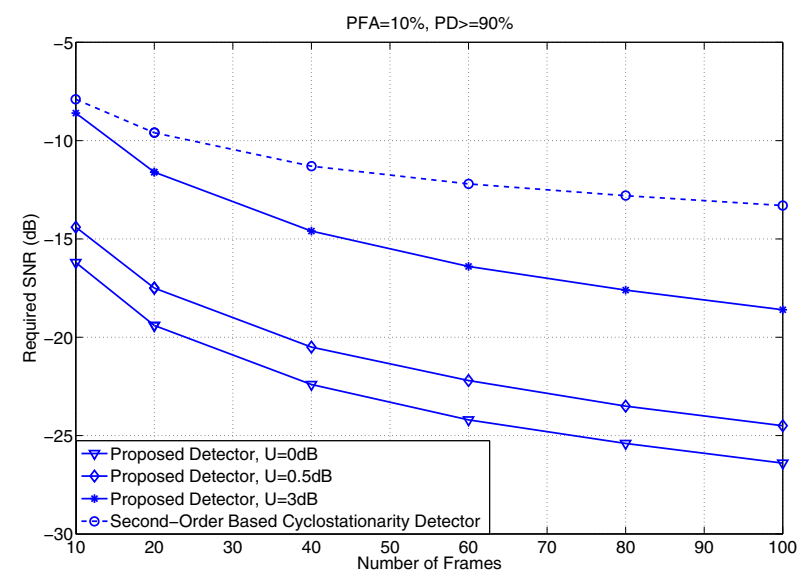

Fig. 9. SNR requirement for successful detection of the primary signal under different observation time for the proposed detector and the secondorder based cyclostatinarity detector

\section{ACKNOWLEDGEMENT}

This work was performed in the framework of EU-ICT WHERE project.

\section{REFERENCES}

[1] A. Ghasemi and E. S. Sousa, "Spectrum sensing in cognitive radio networks: requirements, challenges and design trade-offs", IEEE Commun. Mag., vol. 46, pp. 32-39, Apr. 2008.

[2] Y. Zeng, Y. Liang, A. Hoang, and R. Zhang, "A review on spectrum sensing for cognitive radio: challenges and solutions", EURASIP J. Adv. Signal Process., vol. 2010, 2010.

[3] J. Ma, G. Y. Li, and B. H. Juang, "Signal processing in cognitive radio", Proceedings of the IEEE, vol. 97, no. 5, May 2009.

[4] A. Ghasemi and E. S. Sousa, "Collaborative spectrum sensing for oppotunistic access in fading environments", in Proc. IEEE DYSPAN, Nov. 2005, pp. 131-136.

[5] H.Urkowitz, "Energy detection of unknown deterministic signals", Proc. of the IEEE, vol. 55, no. 4, pp. 523-531, April 1967.

[6] W. A. Gardner, "Exploitation of spectral redundancy in cyclostationary signals", IEEE Signal Process. Mag, vol. 8, pp. 14-36, Apr. 1991. 
[7] R. Tandra and A. Sahai, "SNR walls for signal detection", IEEE Journal on Selected Topics in Singal Processing, vol. 2, pp. 4-17, Feb. 2008.

[8] W. A. Gardner, A. Napolitano, and L. Paura, "Cyclostationarity: Half a century of research", Signal Processing (Elsevier), vol. 86, no. 4, pp. 639-697, 2006.

[9] D. Cabric, S. M. Mishra, and R. W. Brodersen, "Implementation issues in spectrum sensing for cognitive radio", in Proc. Asilomar Conf. Signals, Syst., Comput., Nov. 2004, vol. 1, pp. 772-776.

[10] Y. Zeng and Y. C. Liang, "Maximum-minimum eigenvalue detection for cognitive radio", in Proc. IEEE 18th Int. Symp. PIMRC, Sep. 2007, pp. 145.1-145.5.

[11] Z. Tian and G. B. Giannakis, "A wavelet aprroach to wideband spectrum sensing for cognitive radios", in Proc. of Intl. Conf. on CrownCom, Jun. 2006.

[12] O. A. Dobre, S. Rajan, and R. Inkol, "Joint signal detection and classification based on first-order cyclostationarity for cognitive radios", EURASIP J. Adv. Signal Process., vol. 2009, 2009.

[13] Y. Zhou, K. Qaraqe, E. Serpedin, and O. Dobre, "Am-signal detection in cognitive radios using first-order cyclostationarity", in Proc. IEEE ICASSP, 2010.

[14] A. Sonnenschein and P. M. Fishman, "Radiometric detection of spreadspectrum signals in noise of uncertain power", IEEE Trans. Aerosp. Electron. Syst., vol. 28, pp. 654-660, Jul. 1992.

[15] F. F. Digham, M. S. Alouini, and M. K. Simon, "On the energy detection of unknown signals over fading channels", in Proc. IEEE Int. Conf. Commun., May 2003, pp. 3575-3579.

[16] IEEE Std 802.11g-2003, http://www.ieee.org/, 2003

[17] IST-2003-507581 WINNER D5.4 ver 1.4: Final report on link level and system level channel Models, [Online] http://www.istwinner.org/DeliverableDocuments/D5.4.pdf, Nov. 2005.

[18] A. V. Dandawate and G. B. Giannakis, "Statistical tests for presence of cyclostationary", IEEE Trans. Signal Proceesing, vol. 42, no. 9, pp. $2355-2369,1994$. 